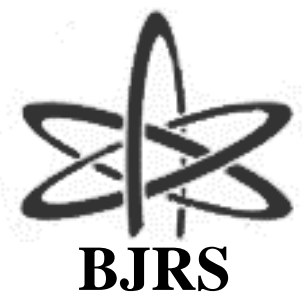

\author{
BRAZILIAN JOURNAL \\ $\mathrm{OF}$ \\ RADIATION SCIENCES \\ 06-02 (2018) 01-15
}

\title{
Analysis in organs at risk for gynecological radiotherapy by high dose rate brachytherapy using Manchester point A,
} ICRU 38/89 Report and American Brachytherapy Society

\section{Recommendation}

\author{
G. Piriz ${ }^{\mathrm{a}}$, Y. Banguero ${ }^{\mathrm{b}, \mathrm{c}}$, A. De Rosa ${ }^{\mathrm{a}}$, C. Vega ${ }^{\mathrm{d}}$ and Aldo Quarneti $^{\mathrm{a}}$ \\ ${ }^{a}$ Instituto de Radiología y Centro de Lucha Contra el Cáncer, Hospital Pereira Rossell, 11600 Montevideo,Uruguay \\ ${ }^{b}$ Centro de Investigaciones Nucleares, Facultad de Ciencias, UdelaR, 11400 Montevideo, Mvd, Uruguay \\ ${ }^{c}$ Cátedra de Radioterapia, Hospital de Clinicas, Fmed, UdelaR, 11600 Montevideo, Mvd, Uruguay \\ ${ }^{d}$ Instituto de Física, Facultad de Ciencias, UdelaR, 11400 Montevideo, Mvd, Uruguay \\ ghpiriz@gmail.com
}

\begin{abstract}
The gynecological treatment with High Dose Rate (HDR) Brachytherapy implies delivering dose to the tumor and spare the dose in organs at risk. In this work, we apply the recommendations for prescribing dose given by the International Commission of Radiation Units (ICRU) reports 38, 89 and the American Brachytherapy Society (ABS). With both schemes of optimization, recommendation of ICRU 38 or ABS with vaginal point from ICRU 89. Doses received by the organs at risk were analyzws, in this case rectum and bladder and also the irradiated volume for patients that receive HDR brachytherapy treatment. An afterloading technique is applied with an Eckert \& Ziegler MultiSource ${ }^{\circledR}$ equipment using a ${ }^{60} \mathrm{Co}$ source, 30 patients with 4 applications with cervical cancer are planning with HDR brachytherapy. This work analyzes 120 single applications with orthogonal images using the treatment planning system (TPS) HDRplus version 3.0.4 through "Auto dwell time determination" optimization method, using the recommendation for optimization dose from the ABS and vaginal point from ICRU 89 . The volume of the isodose curve of prescription is $15 \%$ less using ABS recommendation than ICRU 38. The bladder dose is $11 \%$ and rectum dose is $21 \%$ less using ABS recommendation than ICRU 38. The analysis of dose prescription using ICRU 38 and ABS recommendations in the patients analyzed shows less irradiated volume at the dose prescribed and also less mean dose in rectum and bladder of applications using ABS in contrast with ICRU 38 recommendations.
\end{abstract}

Keywords: ICRU 38, Brachytherapy, HDR. 


\section{INTRODUCTION}

Radiotherapy as one option of treatment for cancer, intending to treat the malignancy determining a target volume and prescribing a dose for that volume depending of the cancer pathology and stadium. It is divided into teletherapy and brachytherapy, determined by the radiation source distance, far or near the patient respectively. In the form of teletherapy, brachytherapy or both, radiation therapy constitutes an integral part of the treatment of carcinoma cervix [1], and most of the radical cases are treated by combination of both modalities [2].

The primary disadvantage of HDR is the potential late toxicity of large dose per fraction. Intracavitary brachytherapy (ICBT) represents an essential component of radiation therapy in carcinoma of the cervix $[3,4]$.

Tod and Meredith [5] outlined the significance of point A, in cancer cervix in 1938. Since then, point A has been widely considered as a very important reference point for dose prescriptions during ICBT. The point A exact meaning and their definition have not always been interpreted in the same way in different centers and even in a given center over a period of time. For example, the original definition of the Manchester System found Point A by drawing a line connecting the upper aspects of the vaginal ovoids and measuring $2-\mathrm{cm}$ superior along the tandem from the interception with this line and then 2-cm perpendicular to this in the lateral direction.

Modifications to the positions and outlining of point A in 1953 have been made to allow it to be easily discernable [6]. Since the target volume is impossible to identify in orthogonal radiographs, the original objective of the identification of point A was to determine a geometric point for the prescription of the dose.

Since the dose gradient in brachytherapy is relatively high compared to teletherapy, brachytherapy has a high gradient dose near the source and also the difficulty of defining the specific volume. In this regard, some investigators have expressed concern about the warnings of dose prescriptions of point A, especially in the modern era, which offers opportunities for dose prescriptions in objective geometry based on imaging studies [7-10].

For many years, different brachytherapy systems as Stockolm, Manchester and Paris were used around the world. Currently the most frequently used protocols are the ICRU recommendations 38 and 89, and the protocol released by the American Brachytherapy Society (ABS) and the Groupe 
Européen de Curiethérapie (GEC) with the European Society for Radiotherapy \& Oncology (ESTRO) known as GEC-ESTRO [11,12]

ICRU 38 protocol is based on the reference volume enclosed by the reference isodose surface $[13,14]$. ABS recommendation use a point $H$ and an optimization to define the dose distribution along the tandem [15].

In brachytherapy, each fraction require careful individualized planning due to the geometrical variation of applicators arising from the differences in the anatomy of the patient, variations in packing and position of the patient, keeping the rectal and bladder doses within the acceptable limits [16-24]. This led to several studies of the problems and uncertainties in the report of the dose $[25,26]$, the dose in the organs [27, 28], and the dependence on the variation of the applicator used $[29,30]$.

Traditionally the planning is doing with radiographer images but now many centers are applying 3D images for brachytherapy planning [31, 32]. ABS and ICRU 38 are based on radiographic images. Another important point to take into account is the optimization as an element that helps to enhance the dose distribution.

The present study attempts to analyze the differences in rectum and bladder dose, as well as the size of the volume enclosed for prescribed isodose curve following ICRU 38 and ABS-ICRU 89 recommendations about intracavitary cervix cancer treated with brachytherapy. For the prescription of the treatment plans, the Manchester points (A points) were used according to ICRU 38 and the points recommended by ABS and ICRU 89. The plans were made with orthogonal radiographs that correspond to the lateral view and the anteroposterior view of the patient. We consider point $\mathrm{A}$ as a function of the geometry of the implant, as specified in ICRU 38.

\section{MATERIALS AND METHODS}

The study was carried out in patients with cervical cancer, who have brachytherapy indicated as part of their treatment. Each brachytherapy process has four sessions of irradiation. This work analyzes differences obtained in the planning treatment for dose prescription by two different methods: ICRU 38 report using the point $\mathrm{A}$ and ABS-ICRU 89 using the point $\mathrm{H}$ and the optimization points; both applied to each patient. A number of 120 single applications that 
correspond to 30 patients were analyzed using orthogonal images for planning with the TPS (Treatment Planning System) HDRplus version 3.0.4 through "Auto dwell time determination" optimization method for ABS recommendations and manual method for Manchester - ICRU38 recommendations. The afterloading equipment is an Eckert \& Ziegler MultiSource ${ }^{\circledR}$ that uses the isotope ${ }^{60} \mathrm{Co}$ (Fig. 1).

Figure 1: Afterloading brachytherapy equipment, ${ }^{60}$ Co Eckert \& Ziegler MultiSource ${ }^{\circledR}$

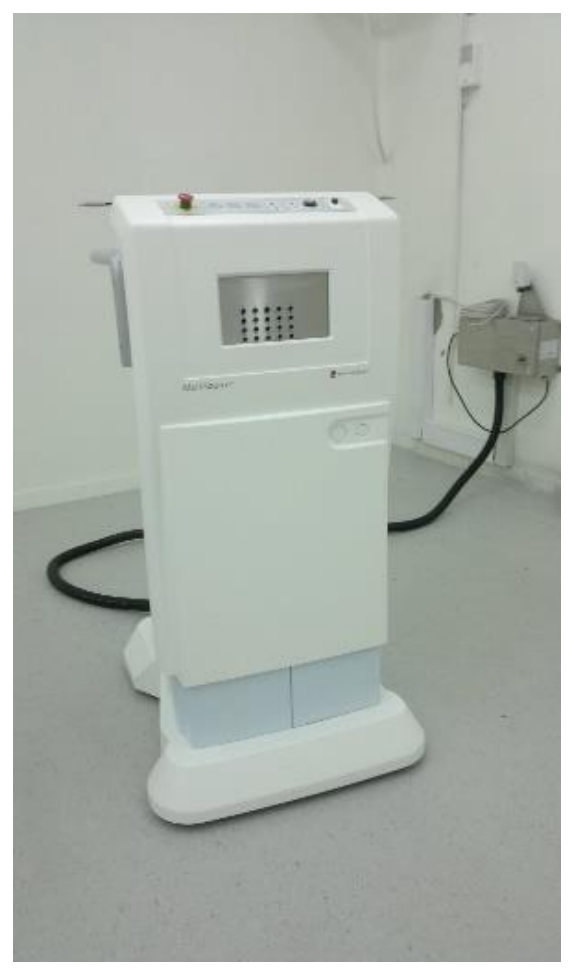

In all cases, procedure in the patient was made with a specific applicator, this was chosen taking into account the patient anatomy and the zone to irradiate. The applicator used was two channel ring applicator and intrauterine tube for ring-applicator with variations in diameter of the ring, long of the intrauterine tube and angle of both applicators (Fig. 2). 
Figure 2: Brachytherapy applicators Ring (above) and Tandem (behind).

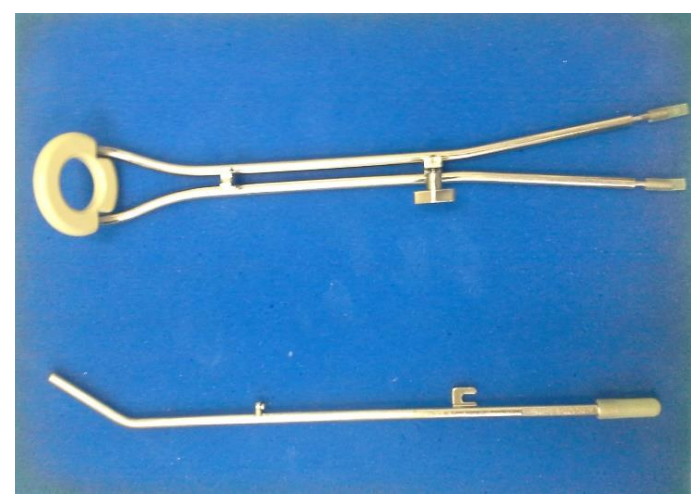

The scheme of intrauterine dispositive inside the patient is shown in Fig. 3 and Fig. 4. The lateral view in Fig 3 is describing the position of the balloon in bladder, and its restriction points; the position of rectum with its restrictions points, and also the dimension indicating the thickness of the prescription isodose t. Figure 4 corresponds to an anteroposterior view showing the dimensions $\mathrm{w}$ (wide) and $\mathrm{h}$ (height) of the prescription isodose; the product of $\mathrm{h}, \mathrm{w}$ and $\mathrm{t}$, results in an estimation of the treated volume.

Figure 3: Schematic anatomical diagram (sagittal view) indicating the isodose of prescription, rectum (rectum point), and balloon in bladder (bladder point). Thickness of the prescription isodose (t) ICRU 38.

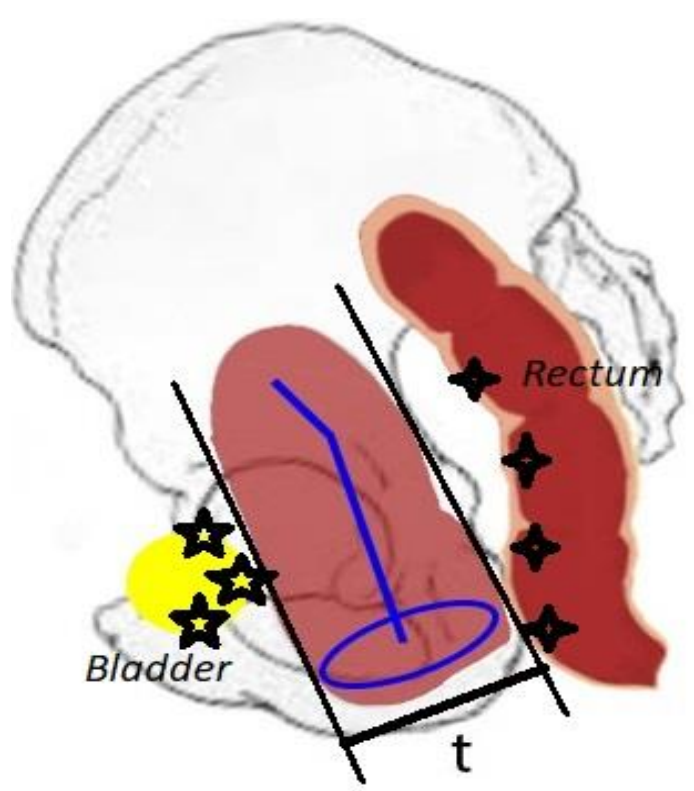


Figure 4: Schematic anatomical diagram (coronal view) indicating the isodose of prescription, height of the prescription isodose (h), and width of the isodose (w) ICRU 38.

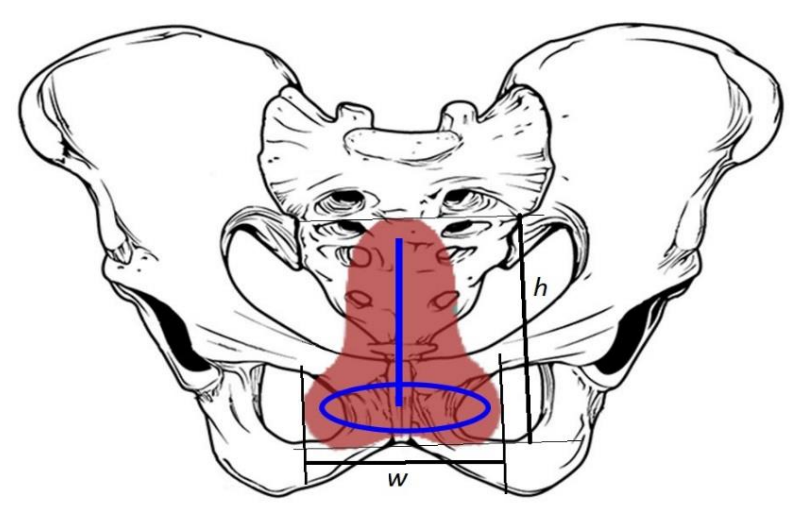

The optimization of the planning was manual using the point $\mathrm{A}$ in the case of ICRU 38, this depends on user criterion. In the case of ABS/ICRU 89 planning, procedure relies on a computational optimization according to the recommendations. It was utilized the points represented in the right of Fig.5 with an additional point on the ring plane with a distance of $1 \mathrm{~cm}$ from the ring source track according to ICRU 89 Report. For this optimization it was used the software planning, setting with auto dwell determination, "Min. /Max. Based Method" and the used parameters are showing in Table 1.

Table 1: Weight and dose for computational optimization of the planning process using ABS recommendation.

\begin{tabular}{ccc}
\cline { 2 - 3 } Parameters & Bladder and Rectum & ABS point \\
\hline Min. Dose $(G y)$ & 0 & 5.8 \\
Min. Weight & 0 & 8 \\
Max. Dose $(G y)$ & 5 & 6.3 \\
Max. Weight & 5 & 8 \\
\hline
\end{tabular}

It was obtained for each patient two planning for every single session of treatment, one for each prescription. Any patient has four treatment sessions. It was considered the average of all four sessions as representative of all treatment for each patient. In consequence, it was obtained two groups to compare for every patient. 
It was calculated the average value for each patient based on several data: $h, t$ and $w$ values, treated volume, media dose in bladder, media dose in rectum, and the volume of the isodose curve that corresponds to the prescribed dose. All this data were used for statistical analysis.

It was applied the t-student test to analyze the data. Assumed as null hypothesis $\mathrm{H} 0$, both groups resulted in equivalent average, therefore H1 assumes that both groups have different media. The significance level of $\mathrm{p}$ is 0.05 . All this analysis process was made using Matlab software.

Figure 5: Representation of the optimization points, the points referenced to the tandem uterine on the right side correspond to the recommendation of the ABS, on the left side the point Manchester A, as defined on ICRU 38/89 report, and at the level of the vaginal ring the point of ICRU 89.

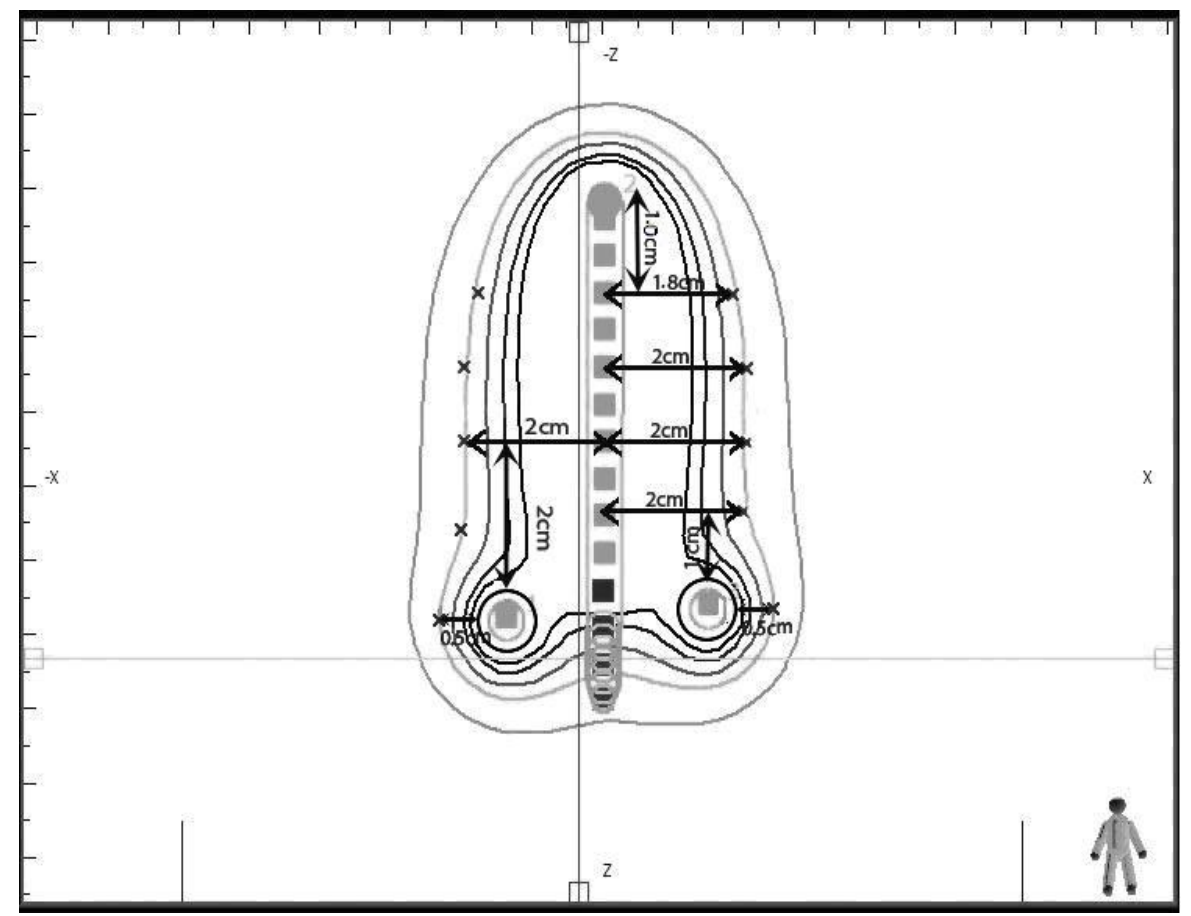

\section{RESULTS AND DISCUSSION}

Table 2 showed the size of dose prescription for both methods, comparing of height the prescription isodose (h) for 30 patients with therapy planning with Manchester-ICRU 38 and ABS recommendation. The average of $\mathrm{h}_{\text {ICRU } 38}=7.7 \mathrm{~cm}$ with $\sigma \mathrm{h}_{\text {ICRU } 38}=0.7 \mathrm{~cm}$ and the average of $\mathrm{h}_{\mathrm{ABS}}=6.5 \mathrm{~cm}$ with $\sigma \mathrm{h}_{\mathrm{ABS}}=0.4 \mathrm{~cm}$; The difference of this distribution $\Delta \mathrm{h}=0.6 \mathrm{~cm}$ with $\mathrm{p}=0.004$ (Fig. 6). 
Table 2 shows the values of $\mathrm{h}, \mathrm{w}$ and $\mathrm{t}$ with its respective standard deviations, for $\mathrm{t}$ both media are the same. Since p was not significant in this value it is not included in the table. Comparing the both planning prescription ICRU 38 has values greater than ABS, the same happen with its respective standard deviations.

Table 2: Comparison of height, width and thickness for both methods.

\begin{tabular}{cccccc} 
& $\mathbf{h}(\mathbf{c m})$ & $\boldsymbol{\sigma h}(\mathbf{c m})$ & $\mathbf{w}(\mathbf{c m})$ & $\boldsymbol{\sigma w}(\mathbf{c m})$ & $\mathbf{t}(\mathbf{c m})$ \\
\hline $\begin{array}{c}\text { Manchester- } \\
\text { ICRU38 }\end{array}$ & 7.7 & 0.7 & 5.4 & 0.2 & 3.9 \\
ABS point & 6.5 & 0.4 & 5.0 & 0.1 & 3.9 \\
\hline
\end{tabular}

Figure 6: Comparison of height the prescription isodose $(h)$.
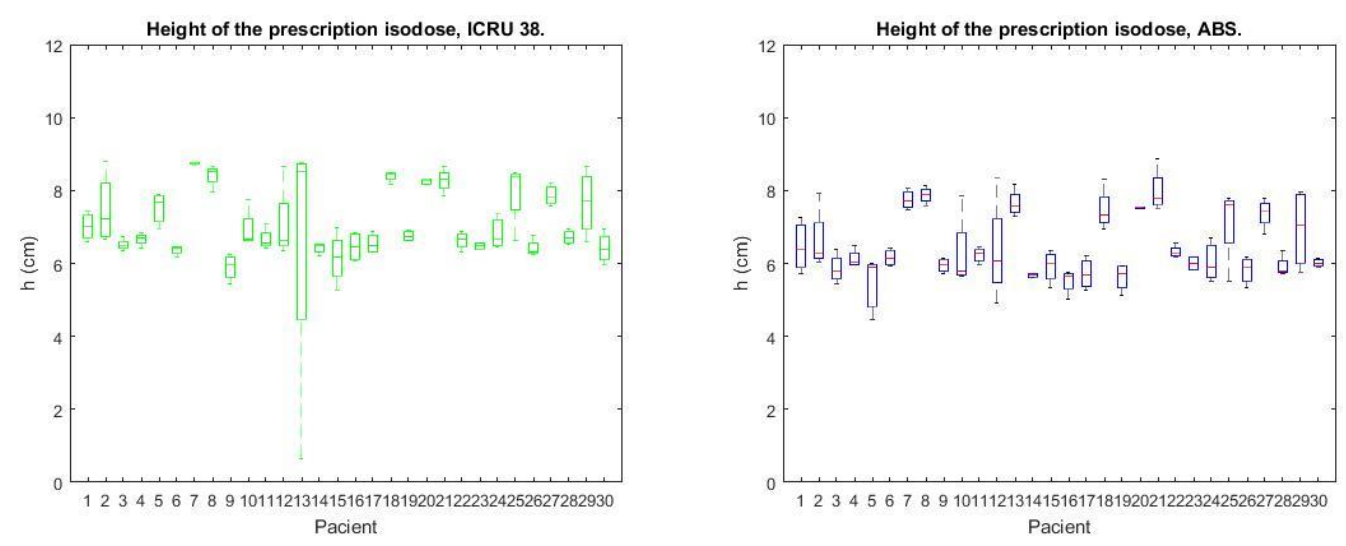

Comparison of width the prescription isodose (w) for 30 patients with therapy planning with ICRU 38 and ABS recommendation. The average of wICRU $38=5.4 \mathrm{~cm}$ with $\sigma \mathrm{wICRU} 38=$ $0.2 \mathrm{~cm}$ and the average of $\mathrm{wABS}=5.0 \mathrm{~cm}$ with $\sigma \mathrm{wABS}=0.1 \mathrm{~cm}$, the difference of this distribution $\Delta \mathrm{w}=0.4 \mathrm{~cm}$ with $\mathrm{p}=0.002$ (Fig. 7). 
Figure 7: Comparison of width the prescription isodose (w).
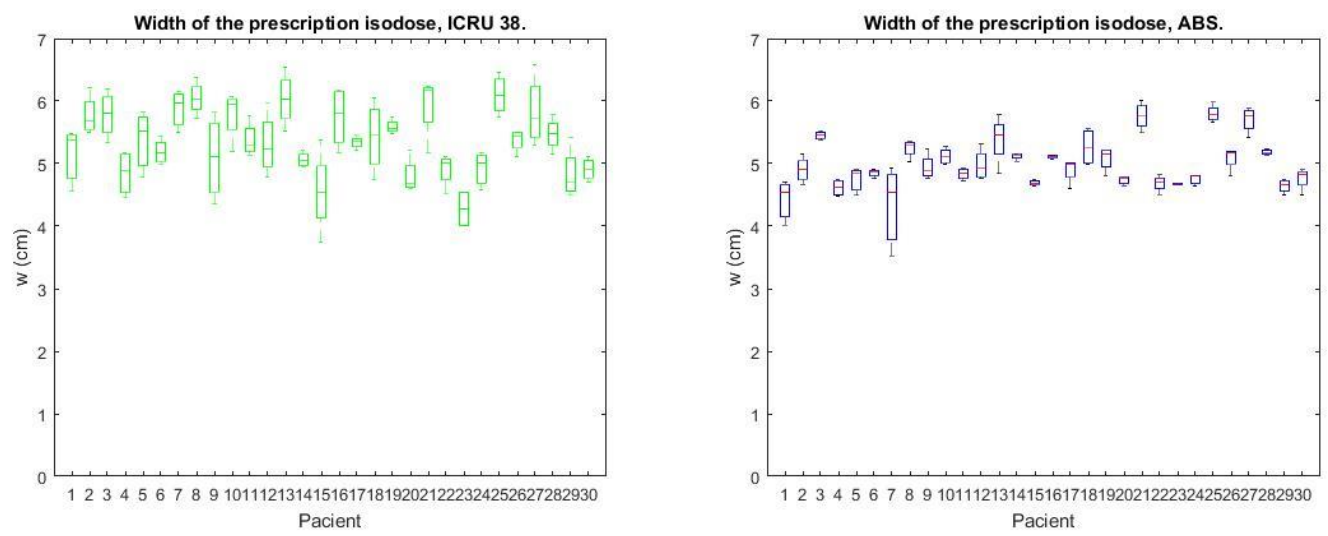

Comparison of thickness the prescription isodose $(\mathrm{t})$ for 30 patients with therapy planning with ICRU 38 and ABS recommendation. The average of $\mathrm{t}_{\text {ICRU }} 38=3.9 \mathrm{~cm}$ with $\sigma \mathrm{t}_{\text {ICRU }} 38=0.2 \mathrm{~cm}$ and the average of $\mathrm{t}_{\mathrm{ABS}}=\mathrm{cm}$ with $\sigma \mathrm{t}_{\mathrm{ABS}}=$, the difference of this distribution is not significant (Fig. 8)

Figure 8: Comparison of thickness the prescription isodose (t).
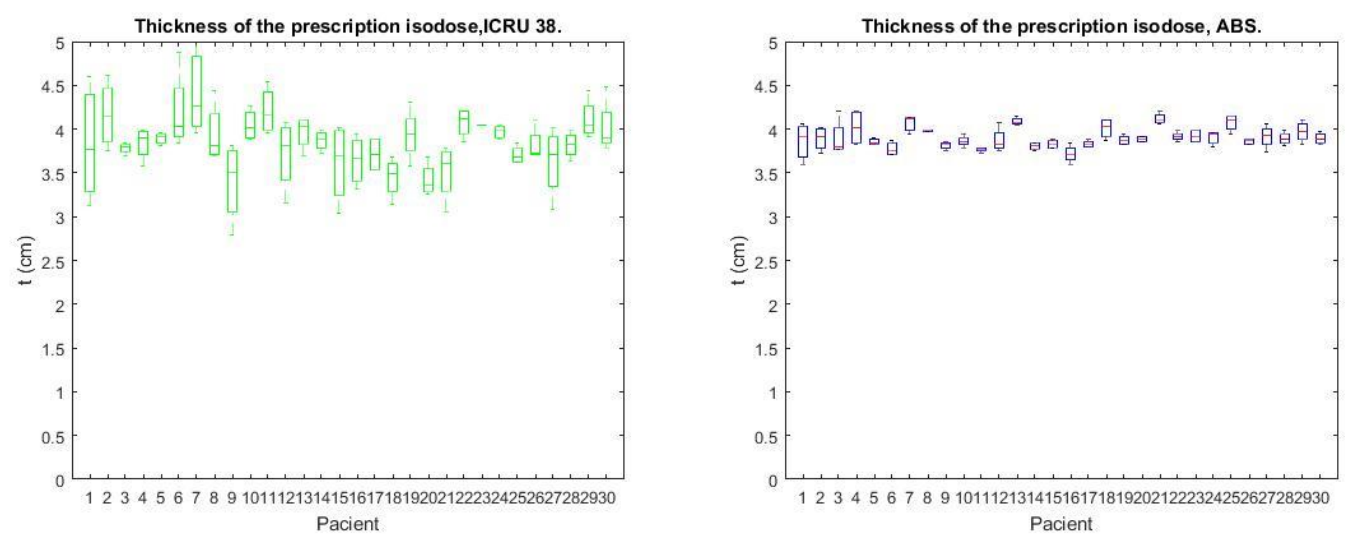

Comparison of $\mathrm{h}^{*} \mathrm{~W} * \mathrm{t}$ the prescription isodose for 30 patients with therapy planning with ICRU 38 and $\mathrm{ABS}$ recommendation. The average of $\mathrm{h} * \mathrm{~W} * \mathrm{t}{ }_{\text {ICRU }} 38=146.7 \mathrm{cc}$ with $\sigma$ hwt $\mathrm{ICRU}_{38}=16.6 \mathrm{cc}$ and the average of $\mathrm{h}^{*} \mathrm{~W}^{*} \mathrm{t} \mathrm{ABS}_{\mathrm{BS}}=126.7 \mathrm{cc}$ with $\sigma \mathrm{hwt} \mathrm{ABS}_{\mathrm{BS}}=9.8 \mathrm{cc}$, the difference of this distribution $\Delta$ $\left(\mathrm{h}^{*} \mathrm{~W} * \mathrm{t}\right)=20.0 \mathrm{cc}$ with $\mathrm{p}=0.003$. Comparison of the volume of the prescription isodose for 30 patients with therapy planning with ICRU 38 and ABS recommendation. The average of VoliCRU 38 
$=78.4$ with $\sigma \mathrm{Vol}_{\mathrm{ICRU}} 38=4.8 \mathrm{cc}$ and the average of $\mathrm{Vol}_{\mathrm{ABS}}=67.2 \mathrm{cc}$ with $\sigma \mathrm{Vol}_{\mathrm{ABS}}=4.4 \mathrm{cc}$, the difference of this distribution $\Delta \mathrm{Vol}=11.2 \mathrm{cc}$ with $\mathrm{p}<0.001$. (Fig. 9)

Figure 9: Comparison of the volume of the prescription isodose

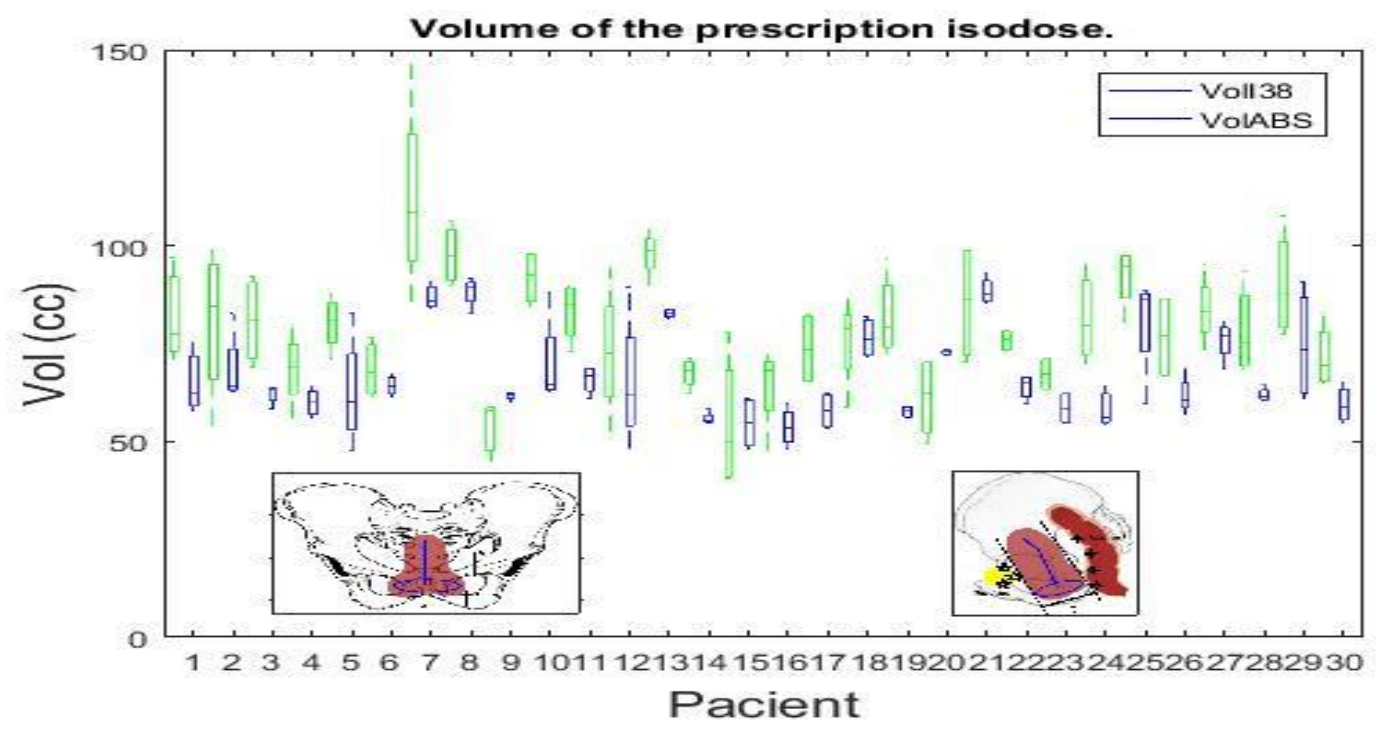

Comparison of the total time for 30 patients with therapy planning with ICRU 38 and ABS recommendation. The average of TotalTime ICRU $38=704 \mathrm{~s}$ with $\sigma$ TotalTime ${ }_{\text {ICRU }} 38=30 \mathrm{~s}$ and the average of TotalTime TBS $=638 \mathrm{~s}$ with $\sigma$ TotalTime $_{\mathrm{ABS}}=29 \mathrm{~s}$, the difference of this distribution $\Delta$ TotalTime $=68 \mathrm{~s}$ with $\mathrm{p}=0.002($ Table 3$)$

Table 3: Comparison of total time for both methods.

Total time (s) $\quad$ ototalTime (s)

Manchester-

704

30

ICRU38

ABS point 638 
For any patient it was studied the size of $\mathrm{t}, \mathrm{w}$ and $\mathrm{h}$ for every treatment session planning using ABS and ICRU 38 recommendations. Figures 6, 7 and 8 show the behavior of $\mathrm{w}, \mathrm{h}$ and $\mathrm{t}$. For $\mathrm{t}$ parameter, the difference is not significance instead of $\mathrm{w}$ and $\mathrm{h}$ that presented significant differences. It is observed that ABS show less average value and variation comparing with ICRU 38. The total volume that correspond to isodose is observed in Figure 9, the difference is significant and for 120 sessions of treatment the average of the product of $\mathrm{h}, \mathrm{w}$ and $\mathrm{t}$ measured is $146.7 \mathrm{cc}$ for ICRU 38, with $\sigma 16.6 \mathrm{cc}$ and 126.7 for ABS, with $\sigma$ 9.8cc. Such parameters presented a significant difference with p-value of 0.003 .

The volume that receive the isodose prescribed is calculated for the planning system and reports for ICRU $3878.4 \mathrm{cc}$ with $\sigma=4.8 \mathrm{cc}$ instead of that for ABS reports $67.2 \mathrm{cc}$ with $\sigma=4.4 \mathrm{cc}$. The difference of that distribution was a $\mathrm{p}<0.001$. This result is shown in Figure 9. The results demonstrated minor size of the volume for ABS

The times of irradiations are smaller (10\%) in the planning using ABS than ICRU 38 recommendation (Table 3).

The volumes built based on 2D images are poor if compared to the ones built with 3D images techniques. For this reason we will start to use 3D images to evaluate the volumes.

Table 4: Dose in rectum for both methods.

\begin{tabular}{ccccccc}
\cline { 2 - 6 } & $\operatorname{Media}(\mathbf{G y})$ & $\boldsymbol{\sigma}_{\text {media }}(\mathbf{G y})$ & $\operatorname{Min}(\mathbf{G y})$ & $\boldsymbol{\sigma}_{\operatorname{Min}}(\mathbf{G y})$ & $\operatorname{Max}(\mathbf{G y})$ & $\boldsymbol{\sigma}_{\operatorname{Max}}(\mathbf{G y})$ \\
\hline $\begin{array}{c}\text { Manchester- } \\
\text { ICRU38 }\end{array}$ & 3.60 & 0.44 & 2.83 & 0,47 & 4.22 & 0.46 \\
ABS point & 3.26 & 0.63 & 2.96 & 0.21 & 3.57 & 0.51 \\
\hline
\end{tabular}

The difference in the media dose is significant with $p=0,0021$, in the same way max dose with $\mathrm{p}<0.01$. 
Table 5: Dose in bladder for both methods.

\begin{tabular}{ccccccc}
\cline { 2 - 5 } & Media $(\mathbf{G y})$ & $\boldsymbol{\sigma}_{\text {media }}(\mathbf{G y})$ & $\operatorname{Min}(\mathbf{G y})$ & $\boldsymbol{\sigma}_{\operatorname{Min}}(\mathbf{G y})$ & $\operatorname{Max}(\mathbf{G y})$ & $\boldsymbol{\sigma}_{\text {Max }}(\mathbf{G y})$ \\
\hline $\begin{array}{c}\text { Manchester- } \\
\text { ICRU38 }\end{array}$ & 2,92 & 0.25 & 2.20 & 0.21 & 3.45 & 0.32 \\
ABS point & 2.64 & 0.32 & 1.87 & 0.16 & 3.20 & 0.56 \\
\hline
\end{tabular}

The results obtained have direct relation with the points used in the process. ICRU 38/Manchester defines just one point, the point A. Consequently optimization is very dependent of the user. In contrast, ABS recommendation suggests an optimization by defining several points, that limit the isodose curve, all of them let to conform easily the dose if it is compared with ICRU 38/Manchester method that gives just one point when performing a prescription and the limit dose of organs at risk.

\section{CONCLUSION}

The ABS recommendation implies optimization using different points along the tandem, this requirement is not in the ICRU 38. Therefore the optimization with ABS allows to obtain a conformed volume around the dispositive in pear form. That is not possible to obtain with ICRU in the same way. This element is crucial and results in a smaller treated volume in ABS than ICRU 38 with less dispersion. The dose received by the bladder and rectum is smaller in ABS than ICRU38. Also values of $\mathrm{h}$ and $\mathrm{w}$ are smaller in ABS recommendation. However the values of $\mathrm{t}$ are similar in both protocols because the constraint of risk organs limited the dose in the same way. The dose in the prescription points is better obtained using the optimization of ABS/ICRU 89.

\section{ACKNOWLEDGMENT}

Thanks to MD Blanca Tazende and Ph. D. Gabriel Gonzalez for all the support. 


\section{REFERENCES}

1. DATTA, N.R.; KUMAR, S.; DAS, K.J.M.; PANDEY, C.M.; HALDER, S.; AYYAGARI, S. Variations of intracavitary applicator geometry during multiple HDR brachytherapy insertions in carcinoma cervix and its influence on reporting as per ICRU report 38. Radiotherapy and Oncology, v. 60, p. 15-24, 2001.

2. ANIL K. T.; KRISHNAM R. A.; DELEEP K. G.; SHABBIR A.; MADHUSUDHANA M. S.; APARNA Y. R. Study of positional dependence of dose to bladder, pelvic wall and rectal points in High-Dose-Rate Brachytherapy in cervical cancer patients. Journal of Medical Physics, v. 38, p. 178-184, 2013.

TALLURI, A. K.; ALLURI KR.; GUDIPUDI D.K.; AHAMED S.; SRESTY M.M.; REDDY A.Y.

3. NORI D.; DASARI N.; ALLBRIGHT R. M. Gynecologic brachytherapy I: proper incorporation of brachytherapy into the current multimodality management of carcinoma of the cervix. Semin Radiat Oncol, v. 12, p. 40-52, 2002.

4. RAJEEV S.; RAHUL B. U.; SARJE M. B.; SINGH K. K. Rectal dosimetry in intracavitary brachytherapy by HDR at rural center of Maharashtra: Comparison of two methods. Journal of Medical Physics, v. 34, p. 93-96, 2009.

5. TOD M.C.; MEREDITH W.J. A dosage system for use in the treatment of cancer of the uterine cervix. Br J Radiol, v. 11, p. 809-823, 1938.

6. TOD M.C.; MEREDITH W.J. Treatment of cancer of the cervix uteri: a revised 'Manchester method'. Br J Radiol, v. 26, p. 252-257, 1953.

7. LEVITT S.H.; KHAN F.M.; POTISH R. Cervical cancer. Levitt \& Tapley's technological basis of radiotherapy: practical clinical applications. 2nd ed., Philadelphia: Lea \& Febiger, 1992. p. 289-298.

8. SCHOEPPEL S.L.; LAVIGNE M.L.; MARTEL M.K. Three dimensional treatment planning of intracavitary gynaecologic implants: analysis of ten cases and implications for dose specification. Int J Radiat Oncol Biol Phys, v. 28, p. 277-283,1994.

9. TERAHARA A.; NAKANO T.; ISHIKAWA A. Dose-volume histogram analysis of high dose rate intracavitary brachytherapy for uterine cervix cancer. Int J Radiat Oncol Biol Phys, v. 35, p. 549-554, 1996.

10. SUYAMA S.; NAKAGUCHI T.; KAWAKAMI K. Computed tomography analysis of causes of local failure in radiotherapy for cervical carcinoma. Cancer, v. 83, p. 1956-1965, 1998.

11. POÈTTERA R.; LIMBERGENB E.V.; GERSTNERA N.; WAMBERSIE A. Survey of the use of the ICRU 38 in recording and reporting cervical cancer brachytherapy. Radiotherapy and Oncology, v. 58, p. 11-18, 2001. 
12. ICRU - International Commission on Radiation Units and Measurements. Dose and volume specification for reporting intracavitary therapy in ginecology, ICRU Report 38, Bethesda: ICRU, 1985. 24p.

13. ICRU - International Commission on Radiation Units and Measurements. Prescribing, Recording, and Reporting Brachytherapy for Cancer of the Cervix, ICRU Report 89, Bethesda: ICRU, 2013. 234p.

14. NAG S.; ERICKSON B.;THOMADSEN B.; ORTON C.; DEMANES J.; PETEREIT D. The American Brachytherapy Society Recommendations for high-dose-rate brachytherapy for carcinoma of the cervix. Int. J. Radiation Oncology Biol. Phys, v. 48, p. 201-211, 2000.

15. DATTA N.; BASU R.; DAS K.; RAJASEKAR D.; PANDEY C.; SINGH U.; AYYAGARI S. Problems and Uncertainties with Multiple Point A's During Multiple High-dose-rate Intracavitary Brachytherapy in Carcinoma of the Cervix. Clinical Oncology, v. 16, p. 129-137, 2004.

16. GARIPAGAOGLU N.; TUNCEL N.G.; DALMAZ M.G.; GULKESEN H.; TOY A.; KIZILDAG A.U. Changes in applicator positions and dose distribution between high dose rate brachytherapy fractions in cervix carcinoma patients receiving definitive radiotherapy. Br J Radiol, v. 79, p. 504-09, 2006.

17. HOSKIN P.J.; COOK M.; BOUSCALE D.; CANSDALE J.; Changes in a€pplicator position with fractionated high dose rate gynecological brachytherapy. Radiother Oncol, v. 40,p. 59-62, 1996.

18. KIM R.Y.; MEYER J.T.; PLOTT W.E.; SPENCER S.A.; MEREDITH R.F.; JENNELLE R.L. Major geometric variations between multiple high-dose rate applications of brachytherapy in cancer of cervix: Frequency and types of variation. Radiology, v. 195,p. 419-22, 1995

19. BAHENA J.H.; MARTINEZ A.; YAN D.; MELE E.; EDMUNSON G.; BROWN D. Spatial reproducibility of the ring and tandem high-dose rate cervix applicator. Int J Radiat Oncol Biol Phys, v. 41, p. 13-9, 1998.

20. HELLEBUST T.P.; DALE E.; SKJONSBERG A.; OLSEN D.R. Inter fraction variations in rectum and bladder volumes and dose distributions during high dose rate brachytherapy treatment of the uterine cervix investigated by repetitive CT examinations. Radiother Oncol, v. 60, p. 273$80,2001$.

21. DATTA N.R.; KUMAR S.; DAS K.J.; PANDEY C.M.; HALDER S.; AYYAGARI S. Variations of intracavitary applicator geometry during multiple HDR brachytherapy insertions in carcinoma cervix and its influence on reporting as per ICRU report 38. Radiother Oncol, v. 60, p. 15-24, 2001.

22. LJUNGGREN L.; WYMAN D.; HARLEY L. An assessment of calculated doses in Intracavitary gynecologic radiotherapy. Med Dosim, v. 12, p. 15-27, 1987. 
23. GRIGSBY P.W.; GEORGIOU A.; WILLIAMSON J.F.; PEREZ C.A. Anatomic variation of gynecologic brachytherapy Prescription points. Int J Radiat Oncol Biol Phys, v. 27, p. 725-9, 1993.

24. SAARNAK A.E.; BOERSMA M.; VAN BUNNINGEN B.N.; WOLTERINK R.; STEGGERDA M.J. Inter-observer variation in delineation of bladder and rectum contours for brachytherapy of cervical cancer. Radiother Oncol, v. 56: p. 37-42, 2000.

25. DATTA N.; BASU R.; DAS K.; RAJASEKAR D.; PANDEY C.; AYYAGARI S. Problems in reporting doses and volumes during multiple high-doserate intracavitary brachytherapy for carcinoma cervix as per ICRU Report 38: a comparative study using flexible and rigid applicators. Gynecologic Oncology, v. 91, p. 285-292, 2003.

26. VINOD S.; CALDWELL K.; LAU K.A.; FO A. A comparison of ICRU point doses and volumetric doses of organs at risk (OARs) in brachytherapy for cervical cancer. Journal of Medical Imaging and Radiation Oncology, v. 55, p. 304-310, 2011.

27. JAMEMA S.; SAJU S.; MAHANTSHETTY U.; PALLAD S.; DESHPANDE D.; SHRIVASTAVA S.; DINSHAW K. Dosimetric evaluation of rectum and bladder using imagebased CT planning and orthogonal radiographs with ICRU 38 recommendations in intracavitary brachytherapy. Journal of Medical Physics, v. 33, p. 3-8, 2008.

28. J. PALVOLGYI, Influence of different Fletchere Suit applicator geometries on sagittal dose distribution. Physica Medica, v. 26, p. 49-54, 2010.

29. DATTAA N.; KUMARA S.; DASA K.; PANDEYB C.; HALDERA S.; AYYAGARIA S. Variations of intracavitary applicator geometry during multiple HDR brachytherapy insertions in carcinoma cervix and its influence on reporting as per ICRU report 38. Radiotherapy and Oncology. v. 60, p. 15-24, 2001.

30. Bahadur Y.A.; Sayed M.E.; Taher Z.H.; Zaza K.O.; Moftah B.A.; Hassouna A.H.; Ghassal N.M.;Using the Computed Tomography in Comparison to the Orthogonal Radiography Based Treatment Planning in High dose Rate (HDR) Brachytherapy in Cervical Uteri Cancer Patients; A Single Institution Feasibility Study. Journal of the Egyptian Nat. Cancer Inst, v. 90, p. 20, 2008.

31. POTTERA R.; HAIE-MEDERB C.; VAN LIMBERGENC E.; BARILLOTD I.; DE BRABANDEREC M.; DIMOPOULOSA J.; DUMASB I.; ERICKSONE B.; LANGA S.; NULENSC A.; PETROWF P.; ROWNDE J.; KIRISITSA C.; Recommendations from gynaecological (GYN) GEC ESTRO working group (II): Concepts and terms in 3D image-based treatment planning in cervix cancer brachytherapy-3D dose volume parameters and aspects of 3D image-based anatomy, radiation physics, radiobiology. Radiotherapy and Oncology, v. 78, p. 6777, 2006.

32. KANNAN R.; GURURAJACHAR J.; PONNI A.; KOUSHIK K.; KUMAR M.; ALVA R.; HARJANI R.; MURTHY A. Comparison of manual and inverse optimization techniques in high dose rate intracavitary brachytherapy of cervical cancer: A dosimetric study, Reports of practical oncology and radiotherapy, v. 20, p. 365-369, 2015. 